\title{
An Efficient Way to Reduce Tag Collision by Using Multidimensional Grouped Backtracking Algorithm
}

\author{
${ }^{1}$ Shilpa Bhatt, ${ }^{2}$ Deepak Dhadwal, ${ }^{3}$ Karishma Bajaj \\ ${ }^{1,2,3}$ (Department of Electronics \& Communication, M.M University, Sadopur, Ambala, Haryana, India) \\ ('Shilpa.arora137@gmail.com, 2deepakdhadwal.007@gmail.com,3vlsi.er86@gmail.com)
}

\begin{abstract}
Radio Frequency Identification (RFID) is a technology which is having the capability of revolutionizing the industry of 'Contact Less Automatic Identification'. The country like ours, has a population whose demands cannot be met unless we catalyst the process of our manufacturing capitals. The process can only be fastened by automating the processes. Machines now days are very well moving towards robotics but inventory management is still an area which needs to be worked upon. A considerably large amount of our medium scale and small scale industries are still dependent on humans for inventory management. Even if it is automated, barcode technology is being practiced at large. Barcode in a sense itself requires manual intervention, thereby, making it prone to errors, and, at the same time is slow. So, to overcome this we can revolutionize in RFID technology, that is indeed a 'Contact Less' Identification. Despite of so many goodies offered by RFID, it is still not practiced by all because of some reasons. One of the major reasons is it is not very economical when considered
\end{abstract} in short term investments.

\section{INTRODUCTION}

Now-a-days, RFID is a developing technology which is being utilized in almost every phase of Industry. It had helped so many businesses, industries, consumer, and factories by cutting down the usage of financial resources \& human resources. The application of RFID has kept on increasing in different sectors in the world [1]. Fig.1.1 shows RFID system depicting its main components -

i. The transponder, which helps in identification of object;

ii. The interrogator, also known as reader, which is used to read or sometimes write \& read both depending on technology \& design used for it.

Almost every company is relying on Auto-ID (Automatic identification) as to get better output in less time. They not only provide correct $\&$ on-time information but also reduced the number of manpower \& errors. These technology can be deployed anywhere to provide necessary information ranging from a small object (living / non-living) to a big Large heavy appliances. The barcode labels results in revolution in auto-ID systems earlier they are being insufficient in an increasing number of cases. Barcodes can be also very cheap, but their stumbling block results in low storage capacity and therefore they cannot be reprogrammed. The solution to this problem is to store data in a silicon chip. The most preferable form of electronic data-based devices used in everyday life is the smart card that is based on its contact field (telephone smart card, bank cards). The mechanical contact that is being used by the smart card is generally impractical. Considering ideal case, using contactless technology, the power which required to operate the electronic data-based devices can be also transferred from the reader. [1].

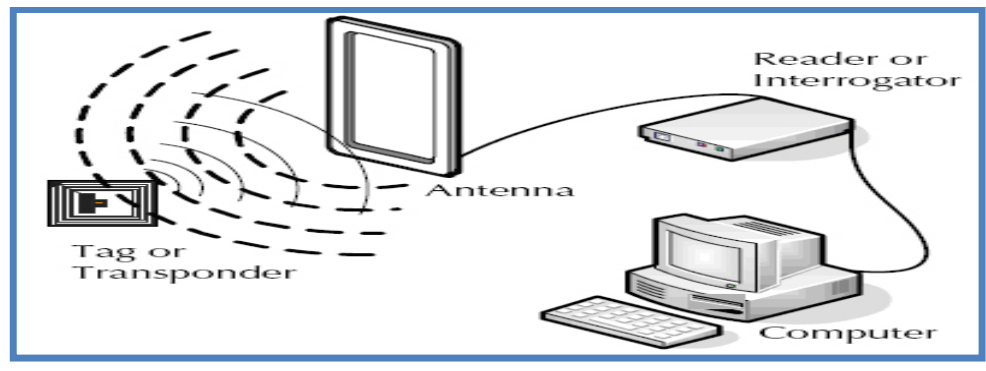

Fig. 1 RFID System Showing Transmission through Tag 


\section{LITERATURE REVIEW}

For handling transmission of frequency with multiple access, previously division multiple access (SDMA) was used. During which associate possibility is to considerably cut back the vary of one reader, however compensation by transportation along an oversized range of readers associated antennas to create an array, so providing coverage of a district. Norman Abrapson, in 1970 [4] planned acknowledgement System for multiple access in radio communications. It works on the principle of your time Division Multiple Access. attributable to the actual fact that at any given time only a fraction of the overall range of consoles within the system are active and since the burst nature of the information from the consoles such a theme can causes the same variety of inefficiencies found in a very wire communication system. In 1979, J. I. Capetanakis [5] invented tree algorithm for packet broadcast of channels, which is again a TDMA type. Each source also responds to a leaf on a binary tree. If there are infinite of sources, then the tree also extends to infinity. This representation of the sources is consider to be a binary addressing scheme. For example, each source has a 4-bit binary address. Also, let $T_{x}$ and $T_{y}$, be two rooted sub trees, and assume that there are no collisions have occurred till the beginning of the present pair of slots. In Binary tree rule it had been ascertained that, at every iterations, the nodes were traversed in same order as in previous form. Thus Wang Jianfang, in 2010 [7] wrote a paper on a completely unique Anti-collision Backtracking rule supported Binary-tree Search. The BBS rule can be implemented as follows, firstly the tags are to be added in the dormant depth counter would like, meantime the reader have to add the collision bit counter and need to jump flag bit. The depth counter dormant will implement the backtracking.

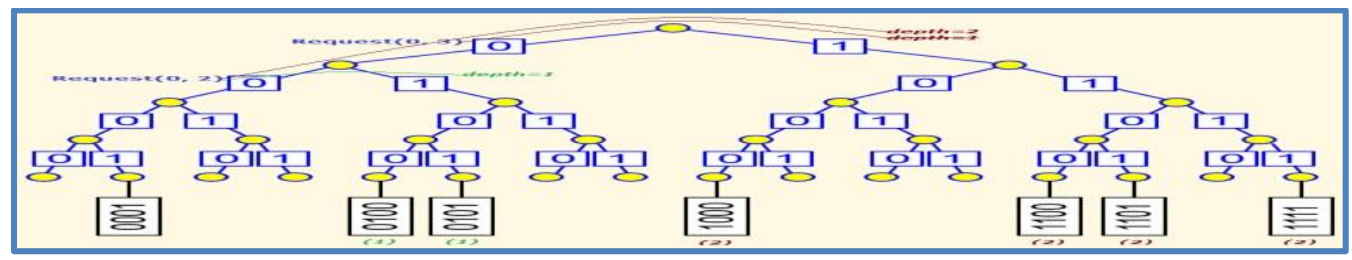

Fig.2: Back Tracking Algorithm

\section{RESEARCH METHODOLOGY AND IMPLEMENTATION}

\section{Multidimensional Grouped Backtracking Binary Search Algorithm}

It is based on the fact that probability of such collisions increases if there is large number of tags in a system. Therefore our aim is to reduce the number of collisions by dividing the groups in small tags. Also, , a multi reader scenario may also be engineered if the tags are wisely divided.

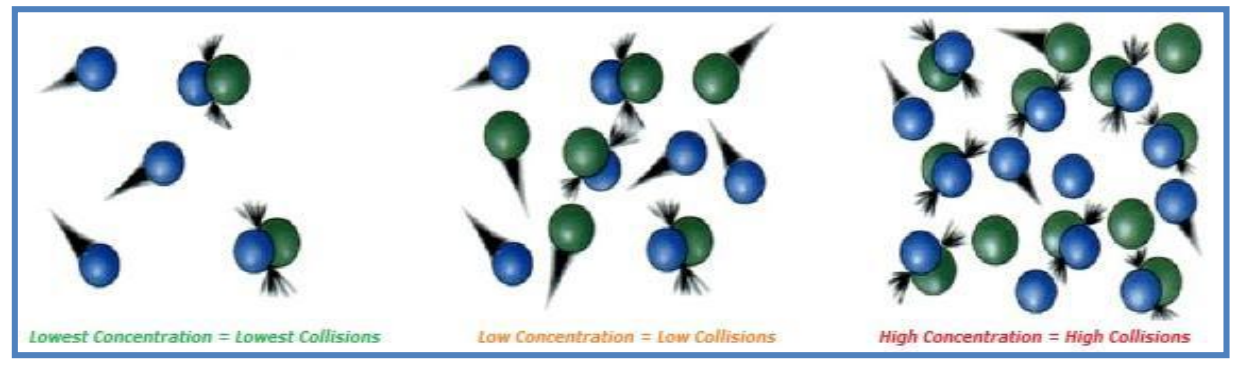

Fig.3: Tag Grouping

This algorithm is basically an extension to - Matrix-based Grouping Method which is based on Anti-collision Algorithm for RFID Tag Identificationll. Therefore this algorithm will use same methodology for grouping of $\mathrm{N}$ tags into $\sqrt{ } \mathrm{N}$ groups as under. [10] 


$\left|\begin{array}{llllll|}\operatorname{tag} 1 & \operatorname{tag} 2 & \operatorname{tag} 3 & \operatorname{tag} 4 & \operatorname{tag} 5 & \operatorname{tag} 6 \\ \operatorname{tag} 7 & \operatorname{tag} 8 & \operatorname{tag} 9 & \operatorname{tag} 10 & \operatorname{tag} 11 & \operatorname{tag} 12 \\ \operatorname{tag} 13 & \operatorname{tag} 14 & \operatorname{tag} 15 & \operatorname{tag} 16 & \operatorname{tag} 17 & \operatorname{tag} 18 \\ \operatorname{tag} 19 & \operatorname{tag} 20 & \operatorname{tag} 21 & \operatorname{tag} 22 & \operatorname{tag} 23 & \operatorname{tag} 24\end{array}\right|$

Fig4: Matrix Grouping

On moving further tags will group themselves into subgroups when the reader sends a second level grouping signal to all the tags in range. The level of sub-division of tags, and, the concept for doing it can be customized based on the nature and properties of application, but for simplicity purpose it may subdivided into two sub-groups, say C \& D, as under. Say each tag is of $\mathrm{k}$ bits. $\mathrm{C}=\{\mathrm{x}: \mathrm{x}$ contains at least $\mathrm{k} / 30$ valued bits $\}, \mathrm{D}=\{\mathrm{x}: \mathrm{x}$ contains less than $\mathrm{k} / 30$ valued bits $\}$, so a multidimensional grouping will be done as under.

Initialize is the first algorithm that is used for reading the tags from excel and dividing it into groups and subgroups with the help of depth matrix.

Inputs: filename - absolute path of excel file containing tag ids

Step 1: Start

Step 2: Set tag_matrix_orig:=xlsread(filename)

Step 3: Initialize tag_matrix_depth array with size equal to that of tag_matrix_orig, and, all the values set to zero Step 4: rows := floor of (square root of (size of tag_matrix_curr))

Step 5: col: $=$ floor of $(($ size of tag_matrix_curr $) /$ rows $)$

Step 6: Initialize status: $=$ false, count: $=-1, \mathrm{i}:=1$

Step 7: Initialize no-one: = floor of (no of bits in tag_matrix_curr(1) / 3)

Step 8: Repeat through Step 16 till i<rows

Step 9: if status $=$ true then increment count by 2 else increment count by 1

Step 10: Set $\mathrm{j}:=1$, status: $=$ false

Step 11: Repeat through step 15 till $\mathrm{j}<\mathrm{col}$

Step 12 Set index: $=((\mathrm{i}-1) * \mathrm{col})+\mathrm{j}$;

Step 13: Set NumberOfOnes: = number of ones in tag_matrix_orig(index)

Step14:if NumberOfOnes>no_one then tag_matrix_depth(index) $:=$ count; else tag_matrix_depth(index) $:=$ count +1 ; status $:=$ true;

Step 15: Increment $\mathrm{j}$ by 1

Step 16: increment i by 1

Step 17: Stop

ReadAll is an algorithm at the top most abstract level. It calls the underlying algorithms till all the tags are read.

Step 1: Start

Step 2: set tag_matrix_curr:=tag_matrix_orig

Step 3: repeat through Step 4 till size (tag_matrix_curr) $>0$

Step 4: Call get Next Iterate Matrix(tag_matrix_curr)

Step 5: Stop

Get Next Iterate Matrix is an algorithm that maintains the depth counter and with the help of another algorithm gets the resolved tag in each iteration

Input: tag_matrix_curr-1D array of all the tags out of which one is to be selected tag_matrix_depth-corresponding current depth counter for each tag 
Output: tag_matrix_curr- 1D array of all the tags but for the one selected in current iteration tag_matrix_depthcorresponding updated depth counter for each tag

Step 1: Start

Step 2: increment all non-zero values in tag_matrix_depth by 1

Step 3: selected tag: = getResolvedTag(tag_matrix_curr)

Step 4: Initialize temp: $=$ zeros $(0,1)$, temp_depth $:=\operatorname{zeros}(0,1)$, is_found $:=0$

Step 5: Repeat through Step 7 till is_found $<1$ and size (tag_matrix_curr) $>1$

Step 6: Set i: $=1$

Step 7: Repeat through Step 10 till i<size (tag_matrix_curr)

Step 8: if tag_matrix_curr $(\mathrm{i})=$ selected_tag then goto Step 10

Step 9: temp(size(temp)+1) $:=$ tag_matrix_curr(i), temp_depth(size(temp_depth)+1) $:=$ tag_matrix_depth(i) -1 If temp_depth(size(temp_depth)+1) $=0$ then set is_found:=1

Step 10: Increment I by 1

Step 11: Set tag_matrix_depth:= temp_depth, tag_matrix_curr:= temp

Step 12: Stop

\section{Simulation of Binary Search Tree}

NRZ encoding is used to simulate binary search shown in table below. It denotes the number of tags read by the reader \& total number of iterations consumed for reading all tags. MATLAB is used which takes the input from excel file and a receiver class generates the corresponding NRZ Code.

Table I Result of Binary Search Algorithm

\begin{tabular}{|l|r|r|r|r|r|} 
Tags & 64 & 128 & 256 & 512 & 1024 \\
\hline Iterations & 192 & 448 & 1024 & 2304 & 5120 \\
\hline
\end{tabular}

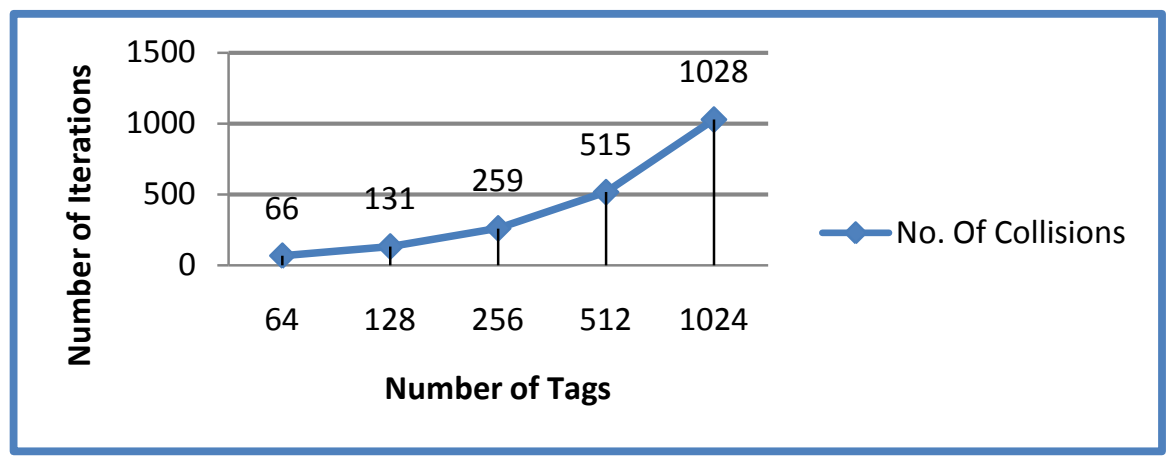

Fig 5: Tag - Iteration Analysis Using Binary Search Algorithm

Table II Result of Back Track Binary Search Tree

\begin{tabular}{|l|l|l|l|l|l|} 
Tags & 64 & 128 & 256 & 512 & 1024 \\
\hline Iterations & 69 & 134 & 263 & 520 & 1033
\end{tabular}




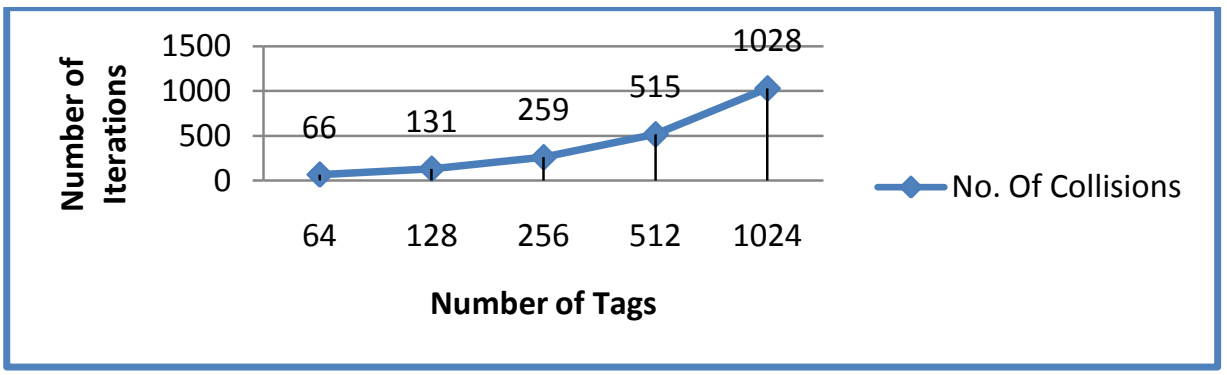

Fig 6: Iteration Analysis Using Back Track Binary Search Tree Algorithm

\section{CONCLUSION AND FUTURE SCOPE}

In this chapter all the methodologies discussed in previous chapters were examined for results. For each methodology individual tables of results were drawn, and, also a table comparing all the results is also drawn. Then using a graph all the three methodologies were examined. Finally the proposed algorithm results were put in table, and, graph for its analysis. It was observed that the results were not significantly improved then that of Matrix based algorithm, but, because of its multi reader capability, a significant improvement can be achieved. The biggest, as of unproven, benefit would ultimately be in supply chain. With rates of simulation such as silicon coming down the cost of reader \& tags will also come down. Once the production of reader \& tag is done at a mass scale their individual cost is bound to come down. With all these factors, it is concluded that RFID is nothing but a leading revolution. RFID technology is increasing day by day in number of industries, the associated privacy and security issues need to be carefully addressed. Because RFID tags come in different flavors, there is no overall, generic RFID security solution. Some low cost basic and passive tags cannot execute standard cryptographic operations like encryption, and hashing. Some tags cost more than basic RFID tags, and can perform symmetric-key cryptographic operations. Organizations are trying to use RFID technology, therefore evaluating the cost and security implications as well as understand the limitations of different RFID technologies and solutions.

\section{REFERENCES}

[1] Klaus Finkenzeller (2010) "RFID Handbook: Fundamentals and Applications in Contactless Smart Cards and Identification ”, John Wiley \& Sons, $3^{\text {rd }}$ Edition, 2010.

[2]Mingsheng Hu, Zhijuan Jia, Xiaoyu Ji and Liu Hong (2013) "RFID Anti-Collision Algorithm Based on Occurrence Position”, Research Journal of Applied Sciences, Engineering and Technology, vol.5, January 2013, pp. 22-29.

[3]Geng Shu-qin, Wu Wu-chen, Hou Li-gang and Zhang Wang (2010) “Anti-Collision Algorithms for Multi-Tag RFID”, Radio Frequency Identification Fundamentals and Applications Bringing Research to Practice, $1^{\text {st }}$ Edition, February 2010.

[4]N. Abramson (1970) “The Aloha System—another Alternative for Computer Communications”, Proc. Fall Joint Computer Conf., Am. Federation of Information Processing Soc. Conf., vol. 37, November 1970, pp.281-285.

[5]J. Capetanakis (1979) “Tree Algorithms for Packet Broadcast Channels”, IEEE Trans. Information Theory, vol. 25, September1979, pp.505-515.

[6] Jihoon Myung, Wonjun Lee, Jaideep Srivastava (2006) “Adaptive Binary Splitting for Efficient RFID Tag Anti-Collision”, IEEE Communications Letters, vol. 10, No. 3, March 2006, pp. 144-146.

[7] Wang Jianfang (2010) “A Novel Anti-collision Backtracking Algorithm Based on Binary-tree Search in UHF”, Proceedings of the Third International Symposium on Computer Science and Computational Technology (ISCSCT), August 2010, pp. 212-214.

[8] Lvqing Yang, Caili Wang, Wenhua Zeng (2013) "Research of Matrix-based Grouping Method on Anti-collision Algorithm for RFID Tag Identification", Advances in information Sciences and Service Sciences (AISS), vol. 5, No. 13, August 2013, pp. 126-123.

[9] Shilpa Bhatt, Deepal Dhadhwal, Ayush Bhatt (2015) "RFID: Review \& Comparison of Tree Based Tag Collision Resolution Algorithms", SSRG International Journal of Electronics and Communication Engineering (SSRG-IJECE), vol. 2, Issue 3, March 2015, pp. 73-77

[10] Haosong Gou, Younghwan Yoo (2012) "Bit Collision Detection Based Query Tree Protocol For Anti Collision In RFID System", International Journal of Innovation Computing, Information \& Control, vol. 8, No. 5(A), May 2012, pp. 3081 - 3102. 
[11] Jihoon Myung, Wonjun Lee, Jaideep Srivastava (2006) “Adaptive Binary Splitting for Efficient RFID Tag Anti- Collision”, IEEE Communications Letters, vol. 10, No. 3, March 2006, pp 144-146.

[12] Parveen Kumar (2013) "Quadratic Search: A New and Fast Searching Algorithm (An extension of classical Binary search strategy)", International Journal of Computer Applications (0975 - 8887), vol. 65, No.14, March 2013, pp

[13] Ajit Singh and Dr. Deepak Garg(2011) “Implementation and Performance Analysis of Exponential Tree Sorting”, International Journal of Computer Applications (0975 - 8887), vol. 24, No.3, June 2011, pp. 34-38.

[14] Ankit R. Chadha, Rishikesh Misal, and Tanaya Mokashi (2014) "Modified Binary Search Algorithm", International Journal of Applied Information Systems (IJAIS, ) vol. 7, No. 2, April 2014.

[15] X.-L. Shi, X.-W. Shi, Q.-L. Huang, and F. Wei (2008) "An enhanced Binary Anti-Collision algorithm of backtracking in RFID System", Progress In Electromagnetics Research, Vol. 4, 2008, pp. 263-271. 